\title{
Frequency Comb Optoacoustic Tomography
}

2

3 Antonios Stylogiannis ${ }^{1}$, Ludwig Prade ${ }^{1}$, Sarah Glasl $^{1}$, Qutaiba Mustafa ${ }^{1}$, Christian Zakian $^{1}$, Vasilis

4 Ntziachristos ${ }^{1, *}$.

5

6 1. Technical University of Munich, Germany, School of Medicine, Chair of Biological Imaging;

7 Helmholtz Zentrum München (GmbH), Neuherberg, Germany, Institute of Biological and Medical

$8 \quad$ Imaging

$9 \quad{ }^{*}$ Corresponding author

10

\section{Key words}

Frequency Domain, Time Domain, Photoacoustics, Laser Diodes, Multispectral, Pulse train

13 


\begin{abstract}
Optoacoustics (OA) is overwhelmingly implemented in the Time Domain (TD) to achieve a high Signalto-Noise-Ratio (SNR). Implementations in the Frequency Domain (FD) have been proposed, but have not offered competitive advantages over TD methods to reach high dissemination. It is therefore commonly believed that the TD represents the optimal way of performing optoacoustics. Here, we introduce a novel optoacoustic concept based on frequency comb and theoretically demonstrate its superiority to the TD. Then, using recent advances in laser diode illumination, we launch Frequency Comb Optoacoustic Tomography (FCOT), at multiple wavelengths, and experimentally demonstrate its advantages over TD methods in phantoms and in-vivo. We demonstrate that FCOT optimizes the SNR of spectral measurements over TD methods by benefiting from signal acquisition in the TD and processing in the FD, and that it reaches the fastest multi-spectral operation ever demonstrated in optoacoustics while reducing performance compromises present in TD systems.
\end{abstract}




\section{Introduction}

30

Generation of optoacoustic (OA) signals requires illumination with energy transients ${ }^{1,2}$. Time Domain (TD) implementations offer large energy transients by means of nanosecond duration light pulses ${ }^{3-6}$, optimizing the signal-to-noise ratio (SNR) and making TD the domain of choice in optoacoustics ${ }^{7-9}$. TD optoacoustic imaging records the time-of-flight of ultrasound waves (US) at multiple locations on the surface of the interrogated object and, using mathematical inversion, converts these measurements to maps of optical absorption ${ }^{10}$.

Other imaging modalities such as optical coherence tomography (OCT) or magnetic resonance imaging (MRI) were originally demonstrated in the TD, but have tremendously benefited from switching operation to the Frequency Domain (FD) ${ }^{11,12}$. The advantages of FD were so fundamental that all OCT or MRI implementations offered today operate in the FD. However, FD-domain optoacoustic implementations using intensity-modulated light ${ }^{13-15}$ provide up to six orders of magnitude ${ }^{16}$ smaller energy transients compared to ultrashort pulses, drastically reducing the SNR ${ }^{17-19}$. Moreover, we have recently shown ${ }^{20}$ the need to capture many discrete frequencies for image generation, which may lead to complex detection (demodulation) schemes ${ }^{20,21}$. Therefore, despite the higher duty cycles achieved over the TD ${ }^{16,20-23}$, FD optoacoustics has offered little impact in the imaging field.

Frequency chirp has also been investigated as a hybrid TD-FD method, by modulating light at a continuously varying frequency ${ }^{22,24}$, thus encoding time in frequency. Detection is carried out in the TD using time-correlation techniques. Similar to FD methods however, the use of sine waves limits the achieved SNR, restricting the use of chirp approaches to experimental investigations.

We propose Frequency Comb (FC) operation to categorically improve upon TD performance, while minimizing FD disadvantages. FC is effectively the reverse implementation of chirp optoacoustics, using a train of discrete pulses, similar to the ones employed in TD optoacoustics, but processing the resulting signals in the FD, representative of an FD system at multiple discrete frequencies ${ }^{20}$. As shown herein, FC illumination allows concurrent illumination at multiple wavelengths without increasing the 
imaging time and yielding an SNR gain that increases by the square root of the number of wavelengths N employed, over TD systems.

Following theoretical considerations, we hypothesized that overdriving laser diodes with FC pulse trains could lead to high quality optoacoustic imaging that could demonstrate benefits over TD implementations. We have shown that overdriven laser diodes yield inexpensive optoacoustic illumination at $>130 \mathrm{~nJ}$ per pulse at repetition rates at the hundreds of $\mathrm{KHz}^{25}$. Based on this illumination ability, we introduce a Frequency Comb Optoacoustic Tomography (FCOT) system (Suppl. Fig.1), implemented with 4 concurrently pulsing wavelengths at $6.8 \mathrm{~ns}$ pulse width, using custom made laser diode drivers and Arbitrary Waveform Generators (AWG). Each wavelength is pulsed at a slightly different repetition rate (see methods). Detection was performed with a $50 \mathrm{MHz}$ central frequency and $110 \%$ bandwidth ultrasound transducer (UST), imparting mesoscopic operation ${ }^{26}$. We show concurrent multi-wavelength imaging of lymphatic and microvascular dynamics in mice at high SNRs, offering the fastest multi-wavelength illumination ever achieved in the field of optoacoustics and confirming spectral performance that improves upon TD implementations.

\section{Frequency Comb Optoacoustics using a single excitation wavelength}

In conventional TD operation, a square light pulse of duration $t_{p}($ Fig.1a) yields a continuous frequency spectrum in the FD, via the Fourier Transform, with the first node at the $1 / t_{p}$ frequency. In FD operation, light modulated by a sine wave in the TD (Fig.1b) yields a single discrete frequency in the FD. FC modulation instead considers a train of pulses (Fig.1c) with a pulse width of $t_{p}$ and a repetition rate $f_{\text {rep }}$. The Fourier Transform of this pulse train yields many discrete frequencies with an envelope (Fig. 1c, right) identical to the continuous spectrum of a single pulse with duration $t_{p}$ (Fig.1a, right). The discrete frequencies of the pulse train are harmonics of the fundamental repetition rate, $f_{\text {rep }}$, i.e. 
integer multiples of $\mathrm{f}_{\text {rep }}$. The frequency spacing and the envelope observed in the FD is determined by the repetition rate of the pulse train (Fig.1d) and the pulse width (Fig.1e) respectively.

Experimental validation of the FC scheme was performed by exciting a black varnish layer on a petri dish at $445 \mathrm{~nm}$, using a pulse train of $t_{p}=6.8 \mathrm{~ns}$ and $\mathrm{f}_{\mathrm{rep}}=200 \mathrm{kHz}$ delivering $189 \mathrm{~nJ}$ per pulse (Fig.1f,g). Averaging in TD (Fig.1g-h) increases the SNR by a factor of sqrt $\left(N_{p}\right)$, where $N_{p}$ is the number of pulses in the pulse train. In contrast, the proposed FC method selects the fundamental frequency $f_{\text {rep }}$ and its harmonics, $k^{*} f_{\text {rep }}$ (Fig.1i) by performing the following operation (see also Suppl. materials):

$$
S_{a}(\omega)=S_{r}(\omega) \sum_{k=1}^{N_{h}} \delta\left(\omega-k \omega_{0}\right),
$$

where $S_{a}(\omega)$ is the Fourier Transform of the averaged signal, $S_{r}(\omega)$ the Fourier Transform of the recorded signal (Fig.1f), $N_{h}$ the number of the harmonics in the recorded bandwidth and $\omega_{0}=2 \pi / T$. This operation selects only the harmonics of $f_{\text {rep }}$ and filters out frequencies that do not contain signal to increase the SNR by the same factor sqrt $\left(N_{p}\right)$ as in the TD (Fig.1j). The signal in Fig.1j is the Fourier Transform of the signal in Fig.1h, with the two signals matching perfectly (Fig.1k). This analysis confirms that FC illumination results in a practical generation of multiple discrete frequencies, required for accurate FD operation, offering an SNR that is equivalent to the TD when a single wavelength is used. Next, we show however that FC offers advantages over TD when multiple wavelengths are employed.

\section{Frequency Comb Optoacoustics using multiple excitation wavelengths}

To demonstrate the FC advantage over the TD (Fig.2) we plotted the single-wavelength excitation pattern in the TD and its power spectrum in Fourier space (Fig.2a) to serve as reference for the analysis that follows. The pulse train shown has a period $T$ that corresponds to a repetition rate $f_{\text {rep }}=1 / T$, a total number of pulses $\mathrm{N}_{\mathrm{p}}$ and an acquisition time $\mathrm{t}_{\mathrm{acq}}=\mathrm{N}_{\mathrm{p}} * \mathrm{~T}$. The period T defines the maximum depth of view DoV $=\mathrm{v}_{\mathrm{s}}{ }^{*} \mathrm{~T}$ that can be achieved for the pulse train selected, where $\mathrm{v}_{\mathrm{s}}$ is the speed of sound.

TD wavelength multiplexing is performed using wavelength interleaving, or time sharing. However, when increasing the number of wavelengths in the TD, at least one of the following three parameters 

each wavelength, or the total acquisition time. Fig.2b shows how the DoV is reduced when using four wavelengths at a given total acquisition time. The different wavelengths excite the tissue using the same repetition rate $f_{\text {rep }}$ but with a time shift $t_{\text {sh }}$ between each wavelength (Fig.2a), given by $t_{s h}=T / N$,

112 where $\mathrm{N}$ is the number of wavelengths. The result is a reduction of the time between subsequent

113 pulses, limiting the DoV available to each wavelength by a factor $\mathrm{N}$. Alternatively, it is possible to

114 retain the original DoV by dropping the repetition rate for each wavelength, $j$, to $f_{\text {rep }, j}=f_{r e p} / N$ and the

115 number of pulses per wavelength to $\mathrm{N}_{\mathrm{p}} / \mathrm{N}$ (Fig.2C), resulting however in an SNR reduction by a factor

116 of sqrt(N). A third alternative, retaining the original DoV and SNR, is to prolong the acquisition time

117 by a factor $\mathrm{N}$ (Fig.2d). 
bioRxiv preprint doi: https://doi.org/10.1101/2021.05.12.443808; this version posted May 14, 2021. The copyright holder for this preprint (which was not certified by peer review) is the author/funder. All rights reserved. No reuse allowed without permission.

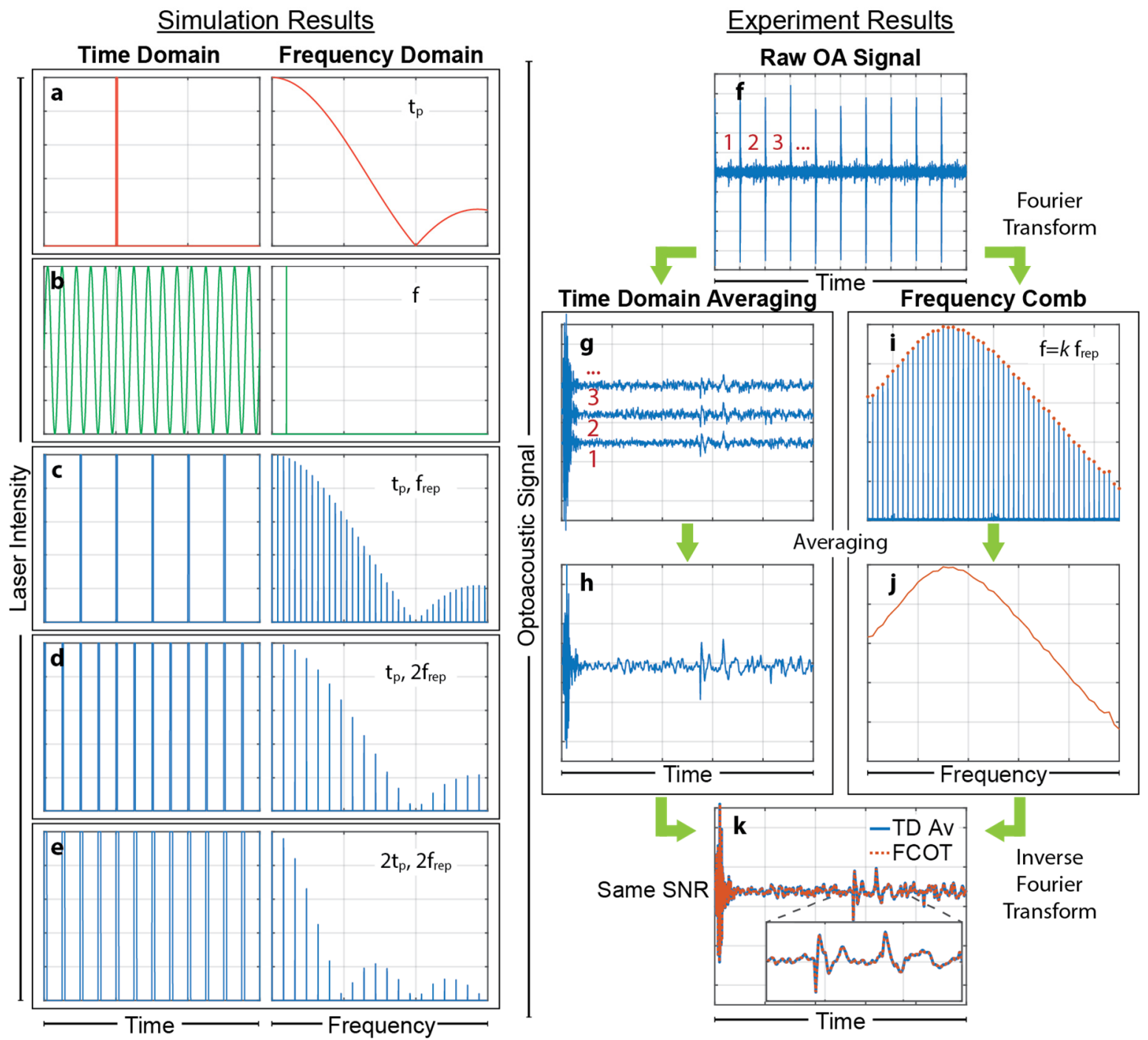

Figure 1. The signal processing algorithms used in Frequency Comb Optoacoustics at a single wavelength. a) A single excitation light pulse of $t_{p}$ duration in TD (left) and a continuous spectrum of frequencies in FD (right). b) $A$ sine wave of frequency $f$ in TD and FD, continuous wave in TD and a single discrete peak in FD. $c$ ) $A$ train of pulses with pulse duration $t_{p}$ and repetition rate $f_{\text {rep }}$ in TD and FD. Many discrete pulses in TD and many discrete frequencies in FD with the same envelope as a single pulse of $t_{p}$ duration in a). d) A train of pulses with pulse duration $t_{p}$ and repetition rate $2 f_{\text {rep }}$ in TD and FD. Twice as many pulses in TD but half the number of discrete frequencies in FD compared to c). e) A train of pulses with pulse duration $2 t_{p}$ and repetition rate $2 f_{\text {rep }}$ in TD and FD. As many pulses in TD and discrete frequencies in FD as in d) but now following a different envelope than a) or c). f) The raw optoacoustic signal recorded using a pulse train like c) for example. $\mathrm{g}$, $\boldsymbol{h}$ ) present the normal averaging in TD. g) The train of pulses is split in sections of period $T=1 / f_{\text {rep }}$ which are averaged (h) point by point. $i, j)$ The Frequency Comb processing of the same signal. i) The Fourier transform of the raw optoacoustic signal (f) with many discrete frequencies that are all harmonics $\left(k^{*} f_{\text {rep }}\right.$ with $k$ positive integer) of the base repetition rate $f_{\text {rep. }}$ In FD we choose only the harmonics of $f_{\text {rep }}$ and discard all the other frequencies that contain only noise (j). k) By performing the inverse Fourier Transform in $\mathbf{j}$ ) we recover the TD signal that matches perfectly with the one in $\boldsymbol{h}$ ). 


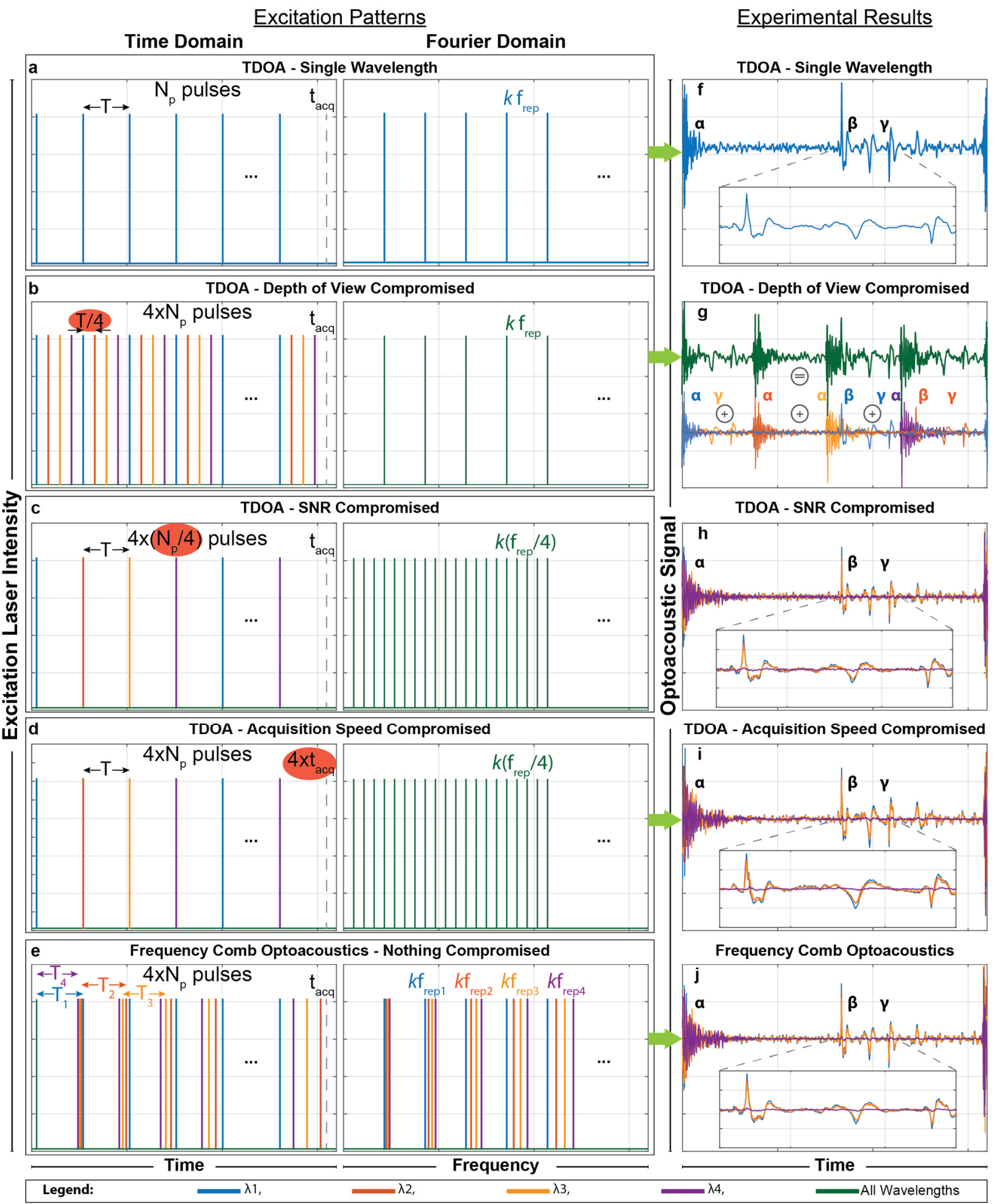

Figure2. FCOT advantages at multiple wavelengths. a) A pulse train of a single wavelength with period T and repetition rate $f_{\text {rep }}=1 / T$, with $N_{p}$ pulses and an acquisition time $t_{a c q}$ as it appears in the time domain (left) and the Fourier domain (right). (b)(d) Multiple wavelengths excitation in TD Optoacoustic. (b) The excitation pattern of 4 wavelengths emitting at the same repetition rate $f_{\text {rep }}$ with a time shift equal to $T / 4, N_{p}$ pulses for all 4 wavelengths and $t_{\text {acq }}$ acquisition time. In this case the depth of view of each wavelength is compromised. (c) The excitation pattern of 4 wavelengths with $f_{\text {rep }} / 4$ repetition rate, $N_{p} / 4$ pulses for each wavelength and $t_{a c q}$ acquisition time. In this case the SNR is compromised. (d) The excitation pattern of 4 wavelengths with $f_{\text {rep }} / 4$ repetition rate, $N_{p}$ pulses per wavelength but with $4 t_{\text {acq }}$ acquisition time. In this case the acquisition time is compromised. (e) Frequency Comb Optoacoustic excitation where all 4 wavelengths have different repetition rates $f_{\text {rep } 1}, f_{\text {rep } 2}, f_{\text {rep } 3}, f_{\text {rep } 4}$ between them, $N_{p}$ pulses for each wavelength and $t_{a c q}$ acquisition time. (f)-(j)The Optoacoustic signal 
recorded by a layer of black varnish on a petri dish from the excitation patterns in (a)-(e) respectively. (f) The optoacoustic signal from a single wavelength. $\boldsymbol{\alpha}$, the electromagnetic interference from the laser diode circuitry when triggered, $\boldsymbol{B}$ the optoacoustic signal from the black varnish, $\boldsymbol{v}$ the reflection of the optoacoustic signal in the petri dish or in the acoustic lens of the UST. (g) The optoacoustic signal from the excitation pattern (b) for all wavelengths (green line) that is the sum of the optoacoustic signal from each wavelength separately (bottom line). The laser interference, optoacoustic signal and its reflections $(\boldsymbol{\alpha}, \boldsymbol{b}, \boldsymbol{v})$ for each wavelength are indicated. The depth of view for each wavelength is drastically reduced. (h) The optoacoustic signal from the excitation pattern (c). The SNR of the optoacoustic signal of each wavelength is reduced. (i) The optoacoustic signal from the excitation pattern (d). The SNR and depth of view of the optoacoustic signal of each wavelength are maintained but the acquisition time is increased. (j) The optoacoustic signal where Frequency Comb Optoacoustic is used. Signal from all 4 wavelengths has been recovered without any cross-talk between the lasers and correctly co-registered in time without compromising depth of view, SNR or acquisition time.

In contrast to TD, FCOT employs a small frequency shift $\delta f$ for the repetition rate of each wavelength

(Fig.2e; time), whereby $\delta f<<f_{\text {rep. }}$ Each wavelength has a different repetition rate which results in a slightly different effective DoV; however, since $\delta f<<f_{\text {rep }}$ this difference is insignificant. The power spectrum of the FCOT excitation pattern (Fig.2e; right) shows the appearance of harmonics from the fundamental repetition rate for each wavelength. The repetition rate of the first laser, $f_{\text {rep, } 1}$, can be selected as the reference repetition rate, with the repetition rate of the remaining lasers given by $f_{\text {rep }, j}=f_{\text {rep }, 1}+(j-1) * \delta f$. FCOT thus recovers the OA signals by evaluating equation (1) with the corresponding $\omega_{0, j}=2 \pi f_{r e p, j}$ for each wavelength. Consequently, using frequency separation, FCOT can multiplex different wavelengths without compromising the DoV, SNR or acquisition time. With the signal processed in the FD, the frequency resolution is defined by $d f=1 / t_{a c q}$, where $t_{a c q}=N_{p} / f_{\text {rep } 1}$, meaning that frequencies that differ by less than $\mathrm{df}$ cannot be resolved. In order to recover the OA signal from all wavelengths, all harmonics from all lasers that lie in the UST detection bandwidth should therefore be spaced at a distance exceeding the frequency resolution $\mathrm{df}$, imposing a minimum number of pulses $N_{p, \text { min }}$ that depends on the UST bandwidth limits ( $f_{\text {low }}$ and $f_{\text {high }}$ ), $N$ and $f_{\text {rep, } 1}$ (see suppl. material). The parameters $N, f_{l o w}, f_{\text {high, }}, f_{r e p, 1}$ and the chosen number of pulses, $N_{p}$, define the range of small frequency shifts $(\delta f)$, between $\delta f_{\min }$ and $\delta f_{\max }$, required to recover the signal of each wavelength without losses. A $\delta f$ value larger than $\delta f_{\min }$ ensures that harmonics of laser $j$ and $j+1$, which lie on the lower end of the UST bandwidth, are well-resolved, while a $\delta f$ value smaller than $\delta f_{\max }$ ensures that harmonics of laser 1 and N, which lie on the upper end of the UST bandwidth, are well-resolved. 
To experimentally demonstrate the advantages of FC operation in multi-wavelength excitation compared to TD optoacoustics, we employed FCOT with four wavelengths at 445, 465, 638 and 808 $\mathrm{nm}$, named wavelengths 1, 2, 3 and 4, respectively. The resulting optoacoustic signal at wavelength $1, f_{\text {rep }, 1}=200 \mathrm{kHz}$ and $N_{p}=200$ (Fig.2f) attained $t_{a c q}=1 \mathrm{~ms}$ and $D o V=7.5 m m$. Electromagnetic interference from the laser diode (LD) driving circuit is indicated with $\alpha$. The primary OA signal generated from black varnish on a petri dish exhibited $21.2 \mathrm{~dB}$ SNR and is indicated with $\beta$, whereby reflections from the UST glass lens that arrive later in time are indicated with $\gamma$.

Fig. $2 \mathrm{~g}$ presents the averaged OA signal in the TD, when the DoV is compromised. Each LD has $f_{\text {rep }, j}=200 K H z, N_{p}=200$ and a time shift between each other that results in $D o V=1.875 \mathrm{~mm}$ and $t_{a c q}=1 \mathrm{~ms}$. The OA signal resulting from the simultaneous excitation using the pattern in Fig. $2 \mathrm{~b}$ is presented as a green line and is the sum of the individual OA signals obtained when each wavelength was pulsed separately (Fig.2g). We could easily detect the laser trigger interference for all wavelengths ( $\alpha$ ). The OA signals from wavelength 1 and $2(\beta)$ are located very closely to the laser interference $(\alpha)$ of wavelengths 3 and 4 respectively, vastly reducing their SNR (5.7dB for wavelength 1$)$. However, the OA signal of wavelength 3 is completely masked by the interference of wavelength 1 . The reflections of the OA signal from wavelength 1,2 and $3(\gamma)$ are still visible. Therefore, electromagnetic interference and OA reflections further limits the DoV and SNR achieved in multiple wavelength TD optoacoustics.

Likewise, SNR limits are imposed (Fig.2h) in response to an excitation pattern (Fig.2c) that uses $f_{\text {rep }, j}=50 \mathrm{kHz}, N_{p}=50$ and $D o V=7.5 \mathrm{~mm}$ for each wavelength with $t_{a c q}=1 \mathrm{~ms}$. For all wavelengths we observed that the laser interference $(\alpha)$, the OA signal $(\beta)$ and the reflections $(\gamma)$ are all visible but with lower SNR (18.8dB for wavelength 1). Finally, with $\mathrm{f}_{\mathrm{rep}, \mathrm{j}}=50 \mathrm{kHz}, \mathrm{N}_{\mathrm{p}}=200$ and $\mathrm{DoV}=7.5 \mathrm{~mm}$ for each laser the OA signal of all 4 lasers can be recorded without SNR losses (21.2dB for wavelength 1$)$ but with $\mathrm{t}_{\mathrm{acq}}=4 \mathrm{~ms}$. 
Conversely, FCOT operation for four wavelengths uses $f_{\text {rep }, 1}=200 \mathrm{kHz}, \delta f=125 \mathrm{~Hz}$ and $N_{p}=200$ and recovers each signal without cross-talk between wavelengths (Fig.2j), experimentally confirming theoretical predictions. FCOT is able to provide high SNR for concurrent excitation with multiple wavelengths, without extending the acquisition time $(1 \mathrm{~ms})$ and achieving the same DoV $(7.5 \mathrm{~mm})$ and SNR (21.2dB for wavelength 1$)$.

We also compared the SNR obtained in conventional FD optoacoustics to FCOT by employing the same black varnish phantom and single wavelength illumination at $445 \mathrm{~nm}$ (see Suppl. FigS1). FD optoacoustics employed a sine wave of $20 \mathrm{MHz}$ frequency with adjusted mean power to equal the mean power output of the FCOT pulsed pattern used for $6.8 \mathrm{~ns}$ pulses at $200 \mathrm{KHz}$. FC demonstrated an SNR that was $20.8 \mathrm{~dB}$ higher compared to FD excitation.

\section{FCOT multi-wavelength imaging of tissues and tissue dynamics in-vivo}

While the theoretical merits of FC optoacoustic operation were demonstrated with phantom measurements, a next critical step was to examine whether FC could offer realistic implementations. For this reason, we aimed to investigate whether the theoretical advantages could lead to operational characteristics (SNR, acquisition speed) that would render FCOT appropriate for in-vivo applications.

A particular unknown parameter in this interrogation was the FCOT performance achieved with multiple wavelengths using laser diodes, as it would be impractical to implement FCOT with multiple solid state lasers due to cost and size. To examine the merits of using low-cost technology, we investigated the performance of multiple laser diodes to image vasculature and lymphatics in-vivo, using the mouse ear as a model. This imaging target was selected as it is a typical tissue where TD optoacoustic implementations have been conventionally demonstrated on in the past. First, we assessed whether FCOT could produce high-quality images from biological specimens. We employed FC illumination at $445 \mathrm{~nm}$ and $465 \mathrm{~nm}$ and resolved oxy- and deoxy-hemoglobin (Fig.3a,b) based on their spectral difference, with deoxygenated hemoglobin absorbing higher at $445 \mathrm{~nm}$, and 
Fig.3b revealed a color-coded composite image (Fig.3c) of the relative vascular oxygenation, with red color corresponding to higher oxygenation levels. We further confirmed that FCOT can produce depth-resolved images (see Suppl. FigS2) without cross-talk between the wavelengths, offering first evidence that FCOT can acquire images from biological tissues based on LDs.

We next aimed to identify whether FCOT could visualize multiple moieties in tissue without compromising operational characteristics as in the TD. We introduced exogenous contrast by intradermal injection of Evan's Blue and Indocyanine Green (ICG) and applied 4-wavelength FCOT to simultaneously resolve arteries and veins (Fig. $3 \mathrm{~d}, \mathrm{e})$ and lymphatic vessels revealed by contrast enhancement (Fig.3f,g). Fig.3h shows the corresponding bright-field image of the mouse ear. The composite image of four wavelengths (Fig.3i) enabled visualization and co-localization of the vascular and lymphatic vessels. Notably, the use of an ultra-wideband transducer enabled to resolve the fine structures represented by the vessels as well as the large absorbing areas that were formed around the injection sites. FCOT can therefore be effectively used to perform OA imaging with multiple wavelengths simultaneously in-vivo with an acquisition of $\sim 30$ mins, while the same multispectral implementation in TD requires $~ 2$ hours.

The FC acquisition acceleration over TD demonstrated in Fig.3 also points to an FCOT use for detecting rapid changes simultaneously using multiple wavelengths. We therefore applied FCOT to monitor oxygenation fluctuations in the mouse ear during an oxygen stress test in-vivo. Fig.4a shows the composite OA image from 2 blue wavelengths revealing the oxygenated and de-oxygenated vessels. We selected a $2 \mathrm{~mm}$ line (Fig. 4a; blue box) to acquire signals repeatedly (continuous FC operation) at a rate of $\sim 4 \mathrm{~Hz}$. A B-scan (indicated by the letter (i) on Fig.4a) revealed an artery (red) and a vein (green) as a function of depth. The oxygen challenge was provided by alternating the composition of the breathing gas from 0.8 liters per minute (lpm) of $100 \% \mathrm{O}_{2}$ (Fig. $4 \mathrm{~b}$; “ $\mathrm{O}_{2}$ ") to $0.6 \mathrm{lpm}$ of $20 \% \mathrm{O}_{2}$ plus 0.2 Ipm $\mathrm{CO}_{2}$ (Fig.4b; "Air"). Fig.4b plots the ratio of the wavelength 2 signal $\left(\mathrm{S}_{2}\right)$ over the wavelength 1 signal $\left(S_{1}\right)$ over time. The $S_{2} / S_{1}$ ratio is indicative of the relative changes of oxygenation in the vessels 

provided. The oxygenation levels in the artery increased faster in response to the change from Air to $\mathrm{O}_{2}$ supply compared to the vein, revealing the expected dynamics of oxygen supply to tissues. The oxygen extraction rate (OER in Fig.4c), an indication of the oxygen uptake by cells, was calculated as $O E R=\left(O_{c a}-O_{c v}\right) / O_{c a}$, whereby $O_{c a}$ and $O_{c v}$ is the oxygen saturation in the central artery and vein, respectively ${ }^{27}$. We observed that OER slightly dropped shortly after the Air supply period, suggesting a delayed response in cell oxygenation. When the oxygen supply was increased during the second and third $\mathrm{O}_{2}$ supply periods, an increased OER was observed followed by a return to normal levels, suggesting that cells consumed more oxygen as it became available. The oxygen stress-test experiment confirms FCOT as a method that can be employed in studying tissue dynamics with high localization ability, confirmed by intravenous injection of ICG and Evan's Blue. Using the same $2 \mathrm{~mm}$ observation field, FCOT recorded contrast agent dynamics at 638 and $808 \mathrm{~nm}$ (Fig.4d). We observed a similar post-injection pattern for both dyes, revealing two distinctive peaks before settling to a baseline value, indicative of the circulation dynamics of the dyes in the vascular system. We further resolved the delayed appearance of the agents in the vein for both dyes, whereby the observed signal increased at a lower rate, compared to the artery, a pattern attributed to dye diffusion in the tissue capillary network. 

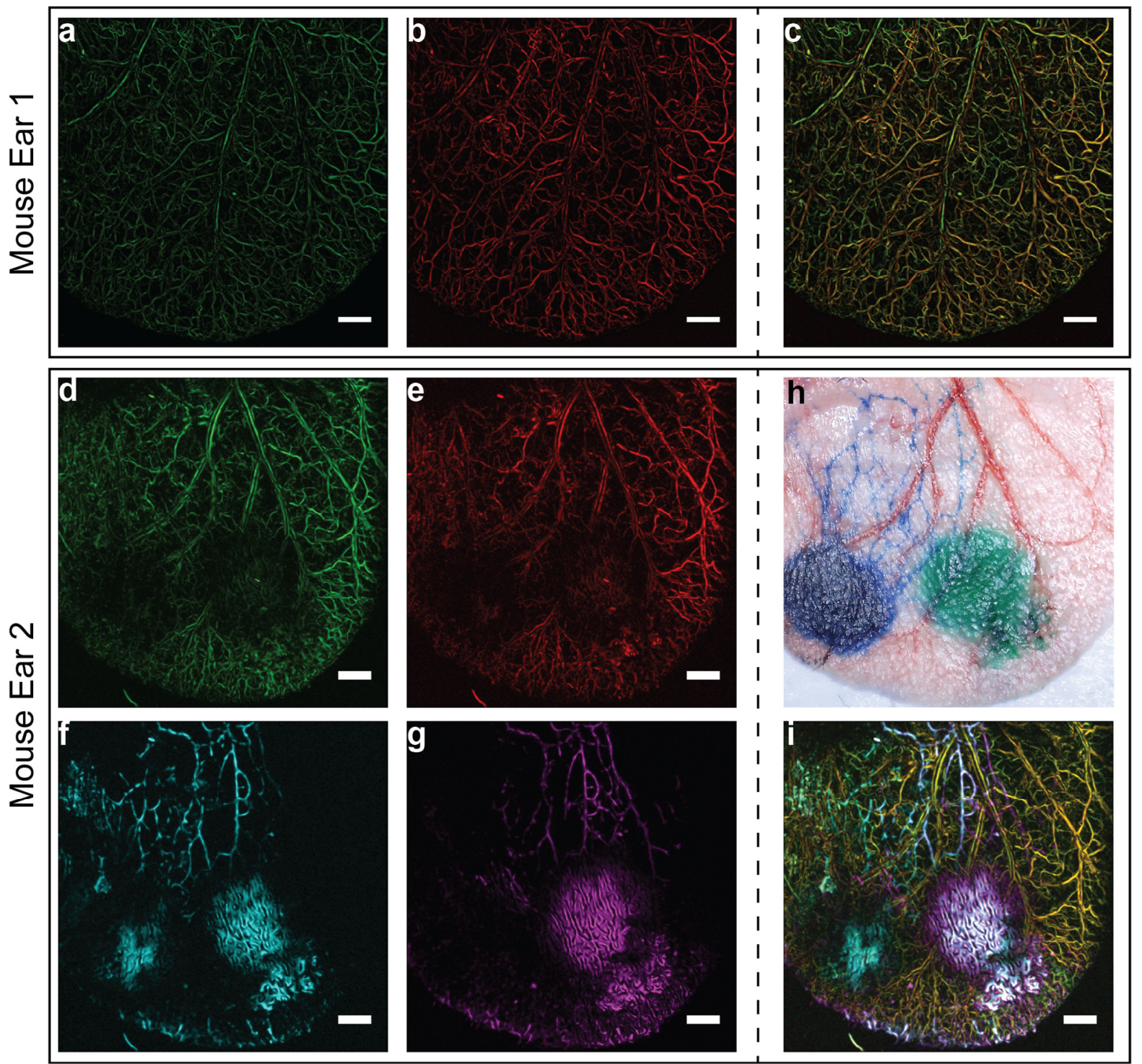

Figure 3. In-vivo imaging using FCOT. $\boldsymbol{a}$ and $\boldsymbol{b}$ present a mouse ear at the 2 wavelengths with high resolution. $c$ shows the composite image color-coded with red indicating higher oxygenation levels compared to green. $\boldsymbol{d}, \boldsymbol{e}, \boldsymbol{g}, \boldsymbol{h}$ presents a second mouse ear at the 4 wavelengths. $f$ a bright field image of the mouse ear. Intradermal injection of Evan's Blue and ICG can be seen in images $\boldsymbol{f}, \boldsymbol{g}, \boldsymbol{h} . \mathbf{g}$ and $\boldsymbol{h}$, the injected dyes enter the lymphatic vessels that present a different structure than blood vessels. i presents the composite image of all 4 wavelengths. We can observe oxygenated (red),de-oxygenated (green) blood vessels and lymphatic vessels after uptake of Evan's Blue (magenta) and ICG (cyan) at the same time. All images are maximum amplitude projections of reconstructed images. Green is $445 \mathrm{~nm}$, Red is $465 \mathrm{~nm}$, Magenta is $638 \mathrm{~nm}$, Cyan is $808 \mathrm{~nm}$, scale bar $1 \mathrm{~mm}$. 

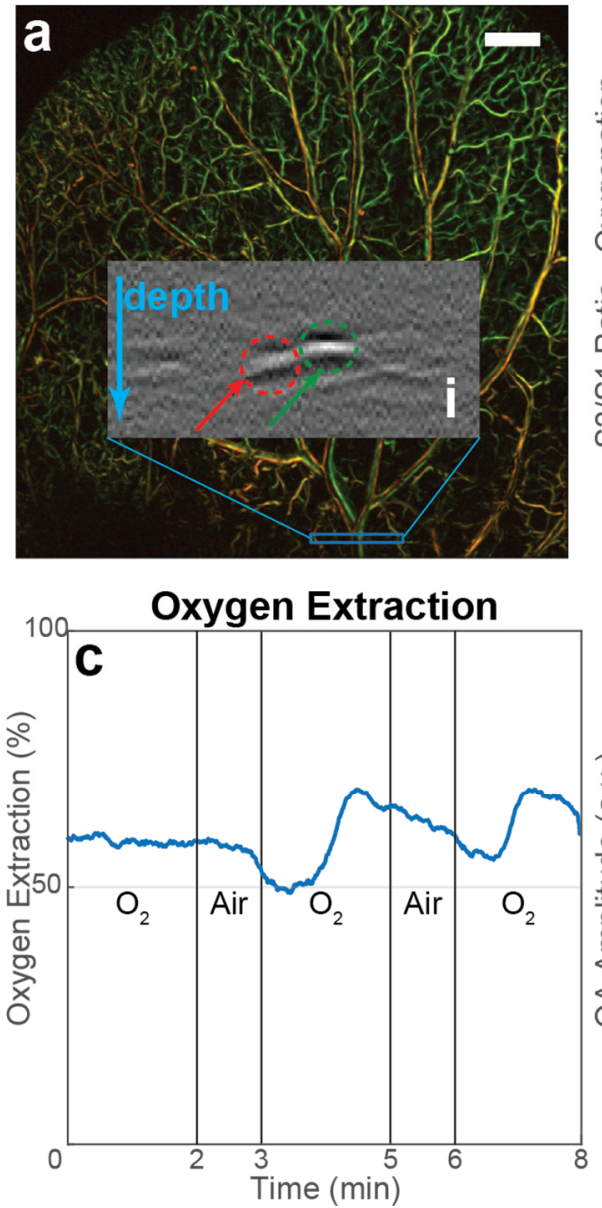

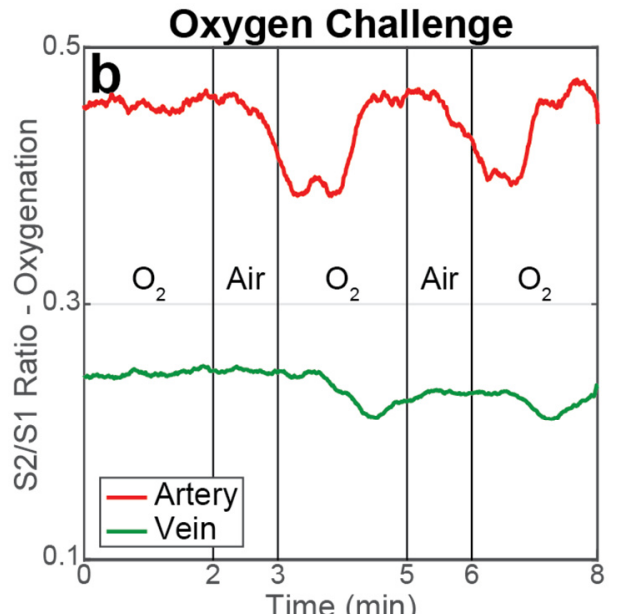

ICG and Evan's Blue Injection

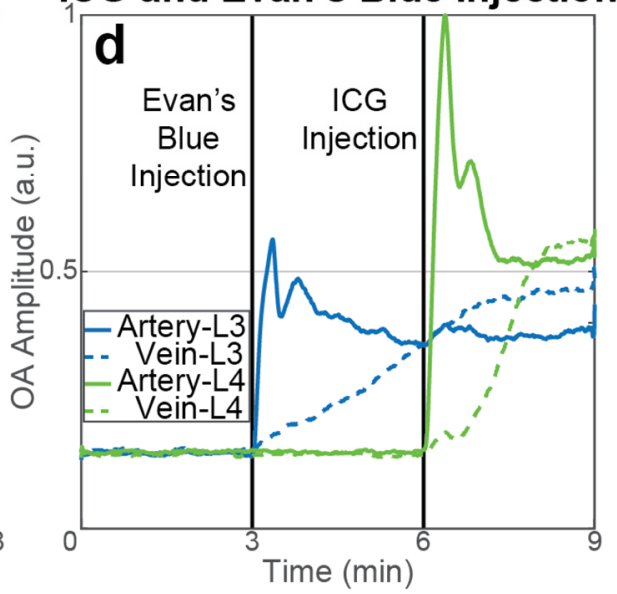

Figure 4. In-vivo vascular dynamics revealed by FCOT. Oxygen challenge experiment and dye injection monitoring in the central vein and artery in the mouse ear using FCOT imaging. Green is 445nm, Red is 465nm, Magenta is 638nm, Cyan is $808 \mathrm{~nm}$, scale bar $1 \mathrm{~mm}$. (a) shows the OA images in the blue wavelengths, showing oxygenated (red) and de-oxygenated (green) vessels. We performed continuously BScans on the blue region denoted in (a) and i shows such a cross-section. The green arrow and region in $\boldsymbol{i}$ indicates the selected vein, and the red arrow and region indicates the selected artery. (b) demonstrates the changes in the ratio between the OA signal intensity in wavelength $2\left(S_{2}\right)$ to that of wavelength $1\left(S_{1}\right)$ in time during an oxygen stress test. The oxygen saturation is proportional to the ratio $S_{2} / S_{1}$. The oxygen saturation changes faster in the artery than in the vein, as expected. (c) shows the oxygen extraction rate during the same experiment. (d) presents the signal intensity at wavelengths 3 and $4\left(S_{3}\right.$ and $S_{4}$ respectively) at the same artery and vein indicated in (a) during intravascular injection of the 2 dyes, Evan's Blue and ICG. In both cases the signal intensity increases first in the artery and later in the vein. 


\section{Discussion}

276

277

278

279

280

281

282

We introduce an optoacoustic method based on the frequency comb concept, which challenges the prevailing notion that TD implementations offer the best optoacoustic performance. By combining the advantages of TD excitation with FD analysis, FCOT offers superior performance to TD at multiwavelength excitation. Increasing the number of wavelengths $(\mathrm{N})$ in TD compromises the depth of view, SNR or the total acquisition time. FCOT can provide simultaneous illumination at multiple wavelengths without sacrificing any of these parameters, enabling either a higher SNR by a factor of sqrt(N) per wavelength, shorter acquisition time by a factor of $\mathrm{N}$, or $\mathrm{N}$ times more depth of view per wavelength compared to TDOA. Moreover, FCOT provides practical implementation of an FD system, by generating multiple discrete frequencies, without the need for individual modulation and demodulation of wavelengths in each of these frequencies. In this way it avoids impractically long acquisition times or complex instrumentation.

A critical aspect herein was not only to show the theoretical superiority of the method, but also to demonstrate that it can lead to practical implementations. An enabling technology that allowed such performance was the use of overdriven continuous wave laser diodes $(C W-L D)^{25}$ which are inherently cost-effective, portable and compact, leading to systems with the potential of high dissemination. Imaging at four wavelengths differentiated blood vasculature from lymphatic vessels in-vivo, reducing the acquisition time from 2 hours required in TD to 30 mins. FCOT also achieved a 4-fold higher acquisition rate compared to TD when performing B-scans over the main artery and vein, enabling the monitoring of relative oxygenation changes and calculation of oxygen extraction rate. Therefore, FCOT is particularly suited for dynamic measurements or for experiments that require anaesthesia, improving also the associated throughput rate over TD implementations. While the maximum number of wavelengths available in FCOT is limited by operational characteristics, up to 28 wavelengths can be simultaneously employed for $f_{r e p, 1}=200 k H z, N_{p}=100$ and $22-78 \mathrm{MHz}$, compared to only 5 wavelengths available to TD implementations with the same operating parameters and a DoV=1.5mm for each wavelength (see Supplementary material). 
302 LDs are ideally suited for FCOT. Besides their small form factor and availability at multiple wavelengths,

303 LDs can be pulsed at very high pulse repetition rates, matching the uniquely optimal FCOT ability to 304 multiplex and average signals, increasing the SNR. New and more powerful LDs entering the market 305 will contribute to further improvements of FCOT performance, offering compact and low-cost systems 306 with high dissemination potential for various applications ${ }^{8,26}$.

307 In summary, FCOT enables for the first time fast, high SNR imaging using multiple wavelengths 308 simultaneously without compromising the DoV and can offer a valuable tool for studying dynamic molecular processes, revolutionizing how multispectral OA imaging will be performed in the future. 


\section{Methods}

\section{Laser Diode Raster Scanning Optoacoustic Tomography System for FCOT}

Supplementary Figure 1 presents the multispectral raster scanning optoacoustic mesoscopy (RSOM) the laser diode drivers and provide a synchronization pulse to the Data Acquisition Card (DAQ, 12-bit, amplified with a 60dB gain amplifier (Miteq AU-1291-R, Miteq, USA) and analog filtered (BLP-90+ and ZFHP-1R2-S+, Minicircuits, USA) before being digitized by the DAQ to avoid aliasing.

The Illumination system consists of $4 \mathrm{CW}$ laser diodes that were overdriven with 4 high-current, shortpulse laser diode drivers developed previously ${ }^{25}$. The laser diodes used in this work are the LDM-4456000 (LaserTack, Germany) emitting at 445nm, the LDM-465-3500 (LaserTack, Germany) emitting at 465nm, the HL63283HG (Ushio, Japan) emitting at 638nm and the K808D02FN (BWT, China) emitting at $808 \mathrm{~nm}$, named laser 1 , laser 2, laser 3 and laser 4 respectively. Each laser diode is focused in a multimode fiber. In order to position each laser diode in a manual X-Y stage (CXY1, Thorlabs, USA), a collimating lens (C340TMD, Thorlabs, USA) is placed in front of it on a manual z-stage (SM1Z, Thorlabs, 
200um fiber core and 0.22NA. One of the outputs is connected to a custom made $95-5 \%$ splitter (LTL the photodiode. The 3 outputs of the power combiner and the $95 \%$ fiber of the splitter were terminated with $1.25 \mathrm{~mm}$ ferrules (SFLC230, Thorlabs, USA) and directed to the Scanning Head. achieve a maximum energy density on the sample.

Scanning and recording is done in a sweeping-like motion using the stages driver as the master in the system. The $x$-stage moves in a straight line with constant velocity. When the $x$-stage has traveled a specific distance, equal to the step size, the stages driver sends a signal to the AWGs to trigger the laser diodes. Each laser diode is triggered using a pulse train with repetition rate $f_{\text {rep } 1}-f_{\text {rep } 4}$ for each laser diode and $\mathrm{N}_{\mathrm{p} 1}-\mathrm{N}_{\mathrm{p} 4}$ number of pulses at each point (A-Scan) in the B-Scan. After the stage has traveled the desired distance, the B-Scan is completed and the stages stop moving. The $y$-stage is moved to the next $y$-position and the x-stage can now perform the next B-Scan in the opposite

direction. For the FCOT excitation we used $f_{\text {rep }, 1}=200000 \mathrm{~Hz}, f_{\text {rep }, 2}=200125 \mathrm{~Hz}, f_{\text {rep }, 3}=200250 \mathrm{~Hz}$ and detailed derivation of these values see Supplementary Material.

To compare the FD OA to FCOT excitation we used a fiber-coupled 450-nm laser diode (FBLD-450$03 \mathrm{HP}$; Picolas, Germany). The output fiber of the laser was pumped into one of the input fibers of the $4 \times 4$ fiber combiner, so that the illumination of the sample is identical for the FD excitation and the 


\section{Reconstruction algorithm}

Image acquisition occurs in a large Field of View $\left(10 \times 10 \mathrm{~mm}^{2}\right)$ and with a scanning step size of 10um

in order to be much lower than the lateral resolution of the system, which is calculated to be 38um.

Time-resolved signals detected at each scanning position correspond to the integration of acoustic processed with a vesselness filter for display ${ }^{30}$. The raw data of a single B-Scan do not allow for 3D image reconstruction. Therefore, a simplified version of the back-projection algorithm was developed, which operates in two dimensions and approximates the transducers sensitivity field as a conic section along the B-Scan direction only.

\section{Laser Diode emission spectra, hemoglobin and dye absorption spectra}

We recorded the emission spectra of the 4 laser diodes used in the multispectral laser diode RSOM

with a spectrometer (USB4000, OceanOptics, UK) and the peak emission wavelengths were 444.3,

$460.1,636.8,804.9 \mathrm{~nm}$ with a variance of $1.6,1.7,1.9,2.2 \mathrm{~nm}$ respectively and an $\mathrm{R}^{2}$ confidence level

higher than 0.96 for all cases.

Hemoglobin has a broad absorption spectrum over the visible and near infrared range with a higher absorption at the lower wavelengths of the spectrum. The absorption of de-oxygenated hemoglobin is higher than the absorption of oxygenated hemoglobin at $444 \mathrm{~nm}$. The absorption of oxygenated hemoglobin is higher than the absorption of de-oxygenated hemoglobin at $460 \mathrm{~nm}$. The total absorption of hemoglobin at wavelengths 1 and 2 is much higher than that at wavelengths 3 and 4 . 
experiments. Moreover, we can estimate the relative changes of oxygen saturation ${ }^{31}$ by calculating the ratio of the signal intensity of wavelength 2 over that of wavelength $1, S_{2} / S_{1}$, with a higher ratio indicating a higher oxygen saturation.

To induce contrast and increase the SNR at wavelengths 3 and 4 we used two dyes, Evan's Blue (Sigma-

this is the only wavelength where we can detect OA signals. ICG in blood plasma has a peak absorption at around $810 \mathrm{~nm}$ and a low absorption at $637 \mathrm{~nm}^{33}$, making it appropriate to induce OA contrast at wavelength 4 with minimal contrast at wavelength 3 and no contrast at the other wavelengths, confirmed by the mouse ear experiments.

\section{Maximum Permissible Exposure (MPE) Limits Compliance}

The energy per pulse on samples was measured with a stabilized thermal power meter (PM160T, Thorlabs, USA) and calculated as 189, 137, 142, $153 \mathrm{~nJ}$ per pulse for lasers 1, 2, 3, 4 respectively. The pulse width was estimated as $6.7,6.7,10.2,10.2$ ns full-width-at-half-maximum (FWHM) for each laser respectively. Using a USB CCD camera (daA1920-30um; Basler AG, Germany) the illumination spot on the surface of the sample was measured to be a circle with a diameter of $\sim 1 \mathrm{~mm}$. Using the step size of 10 um we can calculate the sample exposure.

The total exposure of the sample for simultaneous illumination with all four wavelengths is calculated to be $19.8 \mathrm{uJ} / \mathrm{cm}^{2}$ per pulse and $3.96 \mathrm{~W} / \mathrm{cm}^{2}$ mean exposure, well below the MPE limits of $20 \mathrm{~mJ} / \mathrm{cm}^{2}$ and $18 \mathrm{~W} / \mathrm{cm}^{2}$, imposed by the American National Standards Institute ${ }^{34}$.

\section{Mouse handling and Imaging Protocol}

For our experiments we employed two 5 to 6 -week old female Athymic nude- Foxn $1^{\text {nu }}$ mice (Envigo,

418 Germany). During all measurements, the mice were anesthetized by $1.6 \%$ Isoflurane (cp-Pharma, 

Upper Bavaria, under animal protocol number 55.2-2532.Vet_02-18-120. The first mouse was used for the experiments presented in Fig 3 . The lymphatic ear vessels were highlighted by intradermal administration of $5 \mathrm{ul} \mathrm{ICG}(5 \mathrm{mg} / \mathrm{ml})$ and $5 \mathrm{ul}$ Evan's blue (1\%)into the ear tip of the second mouse. ICG was administered $\sim 30$ min and Evan`s blue $\sim 10$ min before imaging start to ensure the lymphatic drainage of the dyes.

A second mouse was used for the experiments demonstrated in Fig 4. For the oxygen stress experiment, we supplied different isoflurane carrier gas combinations or breathing conditions through a nose mask. The mouse was breathing alternatively $0.8 \mathrm{lpm}$ of $100 \%$ oxygen $\left(\mathrm{O}_{2}\right)$ and a combination of $0.6 \mathrm{lpm}$ medical Air (20\% Oxygen) plus $0.2 \mathrm{lpm}$ carbon dioxide $\mathrm{CO}_{2}$ (Air). For the dye diffusion experiment we initially acquired the background data and after 3 minutes intravenously injected $100 \mathrm{ul}$ of $1 \%$ Evan`s Blue solution and after another 3 minutes $100 \mathrm{ul}$ of the $5 \mathrm{mg} / \mathrm{ml} \mathrm{ICG}$ solution. Both mice were sacrificed immediately after imaging.

\section{Data Availability Statement}

The authors declare that the data supporting the findings of this study are available within the paper and its supplementary information files.

\section{Code Availability Statement}

439 The authors declare that for data collection the commercially available software from GaGe

440 (Dynamic Signals LLC, USA) and Matlab 2016b (Matlab, Mathworks, USA) was used. Data analysis was conducted in Matlab using its built-in functions. The software code is available from the corresponding author upon reasonable request. 


\section{Acknowledgements}

The research leading to these results has received funding by the Bundesministerium für Bildung und Forschung (BMBF), Bonn, Germany (Project Sense4Life, 13N13855), from the European Research Council (ERC) under the European Union's Horizon 2020 research and innovation programme under grant agreement No 694968 (PREMSOT) and from the European Union's Horizon 2020 research and innovation programme under grant agreement No 732720 (ESOTRAC). We would like to thank Dr.

Sergey Sulima for his help writing the manuscript.

\section{$\underline{\text { References }}$}

1. Ntziachristos, V. Going deeper than microscopy: the optical imaging frontier in biology. Nat Meth 7, 603-614 (2010).

2. $\mathrm{Xu}, \mathrm{M}$. \& Wang, L. V. Photoacoustic imaging in biomedicine. Rev. Sci. Instrum. 77, (2006).

3. Diebold, G. J., Sun, T. \& Khan, M. I. Photoacoustic monopole radiation in one, two, and three dimensions. Phys. Rev. Lett. 67, 3384-3387 (1991).

4. Calasso, I. G., Craig, W. \& Diebold, G. J. Photoacoustic point source. Phys. Rev. Lett. 86, 35503553 (2001).

5. Taruttis, A. \& Ntziachristos, V. Advances in real-time multispectral optoacoustic imaging and its applications. Nat. Photonics (2015). doi:10.1038/nphoton.2015.29

6. Brecht, H.-P. et al. Whole-body three-dimensional optoacoustic tomography system for small animals. J. Biomed. Opt. 14, 064007 (2009).

7. Ntziachristos, V. \& Razansky, D. Molecular Imaging by Means of Multispectral Optoacoustic Tomography (MSOT). Chem. Rev. 110, 2783-2794 (2010).

8. Aguirre, J. et al. Precision assessment of label-free psoriasis biomarkers with ultra-broadband optoacoustic mesoscopy. Nat. Biomed. Eng. 1, 1-8 (2017).

9. Omar, M., Schwarz, M., Soliman, D., Symvoulidis, P. \& Ntziachristos, V. Pushing the Optical Imaging Limits of Cancer with Multi-Frequency-Band Raster-Scan Optoacoustic Mesoscopy (RSOM). Neoplasia 17, 208-214 (2015).

10. Rosenthal, A., Ntziachristos, V. \& Razansky, D. Acoustic Inversion in Optoacoustic Tomography: A Review. Curr. Med. Imaging Rev. 9, 318-336 (2013).

11. de Boer, J. F., Leitgeb, R. \& Wojtkowski, M. Twenty-five years of optical coherence tomography: the paradigm shift in sensitivity and speed provided by Fourier domain OCT [Invited]. Biomed. Opt. Express 8, 3248 (2017).

12. Kumar Jhamb, T., Rejathalal, V. \& Govindan, V. K. A Review on Image Reconstruction through MRI k-Space Data. Int. J. Image, Graph. Signal Process. 7, 42-59 (2015).

13. Kellnberger, S., Deliolanis, N. C., Queirós, D., Sergiadis, G. \& Ntziachristos, V. In vivo frequency domain optoacoustic tomography. Opt. Lett. 37, 3423-3425 (2012).

14. Mohajerani, P., Kellnberger, S. \& Ntziachristos, V. Frequency domain optoacoustic tomography using amplitude and phase. Photoacoustics 2, 111-118 (2014).

15. Telenkov, S., Mandelis, A., Lashkari, B. \& Forcht, M. Frequency-domain photothermoacoustics: Alternative imaging modality of biological tissues. J. Appl. Phys. 105, 1-8 (2009). 
16. Maslov, K. \& Wang, L. V. Photoacoustic imaging of biological tissue with intensity-modulated continuous-wave laser. J. Biomed. Opt. 13, 24005-24006 (2008).

17. Langer, G., Buchegger, B., Jacak, J., Klar, T. A. \& Berer, T. Frequency domain photoacoustic and fluorescence microscopy. Biomed. Opt. Express 7, 2692 (2016).

18. Lashkari, B. \& Mandelis, A. Comparison between pulsed laser and frequency-domain photoacoustic modalities: Signal-to-noise ratio, contrast, resolution, and maximum depth detectivity. Rev. Sci. Instrum. 82, (2011).

19. Telenkov, S. \& Mandelis, A. Signal-to-noise analysis of biomedical photoacoustic measurements in time and frequency domains. Rev. Sci. Instrum. 81, 1-7 (2010).

20. Kellnberger, S. et al. Optoacoustic microscopy at multiple discrete frequencies. Light Sci. Appl. 7, (2018).

21. Lathi, B. P., Sedra, A. S. \& Valkenburg, M. E. Van. Modern Digital and Analog Communication Systems. (Oxford University Press, Inc., 1995).

22. Mandelis, A. \& Guo, X. Wavelength-modulated differential photothermal radiometry: Theory and experimental applications to glucose detection in water. Phys. Rev. E-Stat. Nonlinear, Soft Matter Phys. 84, 1-14 (2011).

23. Lashkari, B., Sean Choi, S. S., Khosroshahi, M. E., Dovlo, E. \& Mandelis, A. Simultaneous dualwavelength photoacoustic radar imaging using waveform engineering with mismatched frequency modulated excitation. Opt. Lett. 40, 1145 (2015).

24. Dovlo, E. et al. Quantitative phase-filtered wavelength-modulated differential photoacoustic radar tumor hypoxia imaging toward early cancer detection. J. Biophotonics 10, 1134-1142 (2017).

25. Stylogiannis, A. et al. Continuous wave laser diodes enable fast optoacoustic imaging. Photoacoustics 9, 31-38 (2018).

26. Omar, M., Aguirre, J. \& Ntziachristos, V. Optoacoustic mesoscopy for biomedicine. Nat. Biomed. Eng. 3, 354-370 (2019).

27. Molnar, Z. \& Nemeth, M. Monitoring of tissue oxygenation: An everyday clinical challenge. Front. Med. 4, 1-6 (2017).

28. Kostli, K. P., Frenz, M., Bebie, H. \& Weber, H. P. Temporal backward projection of optoacoustic. Phys. Med. Biol. 46, 1863-1872 (2001).

29. Omar, M. et al. Optical imaging of post-embryonic zebrafish using multi orientation raster scan optoacoustic mesoscopy. Light Sci. Appl. 6, e16186-e16186 (2017).

30. Longo, A. et al. Assessment of hessian-based Frangi vesselness filter in optoacoustic imaging. Photoacoustics 20, 100200 (2020).

31. Sei, K. et al. Measurement of blood-oxygen saturation using a photoacoustic technique in the rabbit hypoxemia model. J. Clin. Monit. Comput. 33, 269-279 (2019).

32. Radu, M. \& Chernoff, J. An in vivo assay to test blood vessel permeability. J. Vis. Exp. 2-5 (2013). doi:10.3791/50062

33. Landsman, M. L. J., Kwant, G., Mook, G. A. \& Zijlstra, W. G. Light absorbing properties, stability, and spectral stabilization of indocyanine green. J. Appl. Physiol. 40, 575-583 (1976).

34. Delori, F. C., Webb, R. H. \& Sliney, D. H. Maximum permissible exposures for ocular safety (ANSI 2000), with emphasis on ophthalmic devices. J. Opt. Soc. Am. A 24, 1250 (2007). 

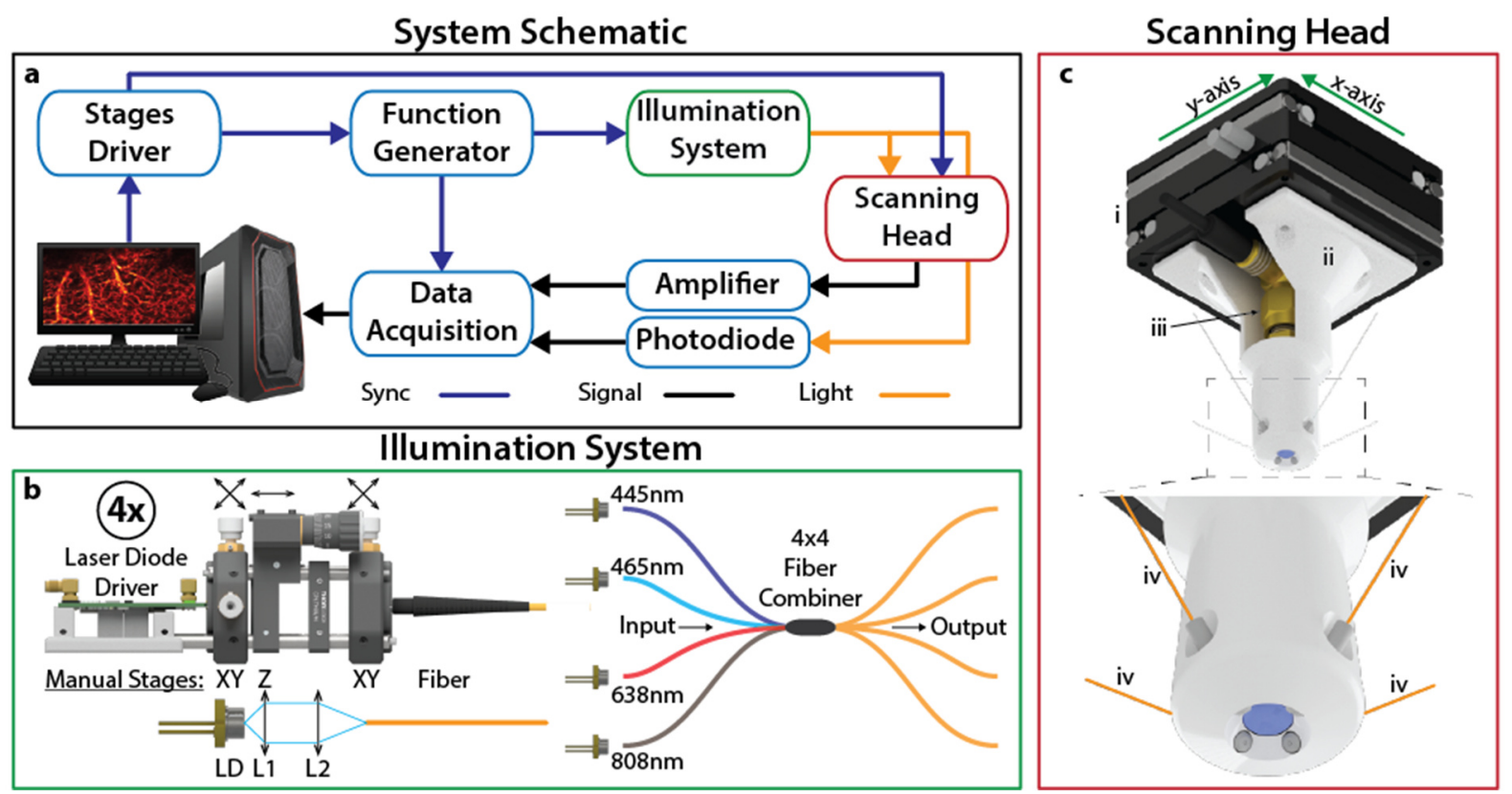

Supplementary Figure1. Multispectral Raster Scanning Optoacoustic Mesoscopy system using 4 laser diodes. a The schematic of the system developed showing the electrical and optical connections of the different parts of the system. $\boldsymbol{b}$ The laser diode illumination system. Each laser diode is attached to a separate laser diode driver and focused into a multimode fiber with a 2 lens system. The 4 laser diodes are coupled in a $4 \times 4$ fiber power combiner and each output of the combiner has $25 \%$ of the power of each input, combining all the wavelengths. $c$ The scanning head of the RSOM system consisting of the $x-y$ scanning stages (i), the 3D printed holder (ii), the UST (iii) and the 4 output fibers of the fiber power combiner (iv) arranged in a circular pattern around the UST. 
bioRxiv preprint doi: https://doi.org/10.1101/2021.05.12.443808; this version posted May 14, 2021. The copyright holder for this preprint (which was not certified by peer review) is the author/funder. All rights reserved. No reuse allowed without permission.

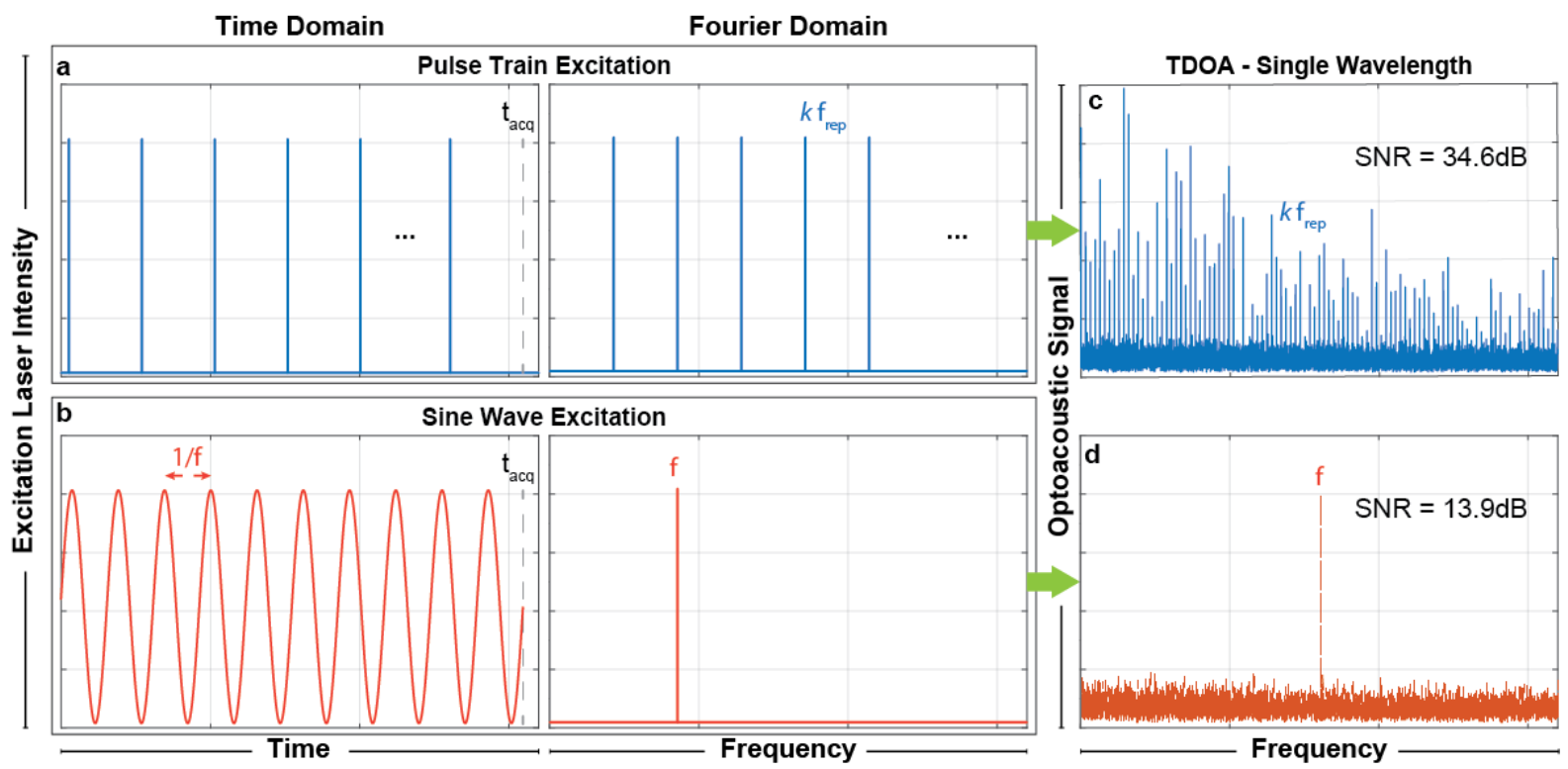

Supplementary Figure2. FCOT advantages over FD-OA at single-wavelength illumination. $a$ The FC pulse train excitation in Time Domain and Fourier space. $\boldsymbol{b}$ The sinusoidal FD excitation at frequency $f$ in Time Domain and Fourier space. $\boldsymbol{c}$ The optoacoustic signal from a black varnish layer on a petri-dish after excitation with the pattern in a in the Fourier space after recording it in time and performing the Fourier Transform. The signal appears as many discrete frequency peaks that are all harmonics of the laser pulse repetition rate $f_{\text {rep. }}$. The total OA signal SNR is calculated to be 34.6dB. $\boldsymbol{d}$ The optoacoustic signal from the sinusoidal excitation in $\boldsymbol{b}$ in Fourier space from the same sample. Now the signal appears in only 1

546 frequency, $f$, with an SNR of $13.9 \mathrm{~dB}$.
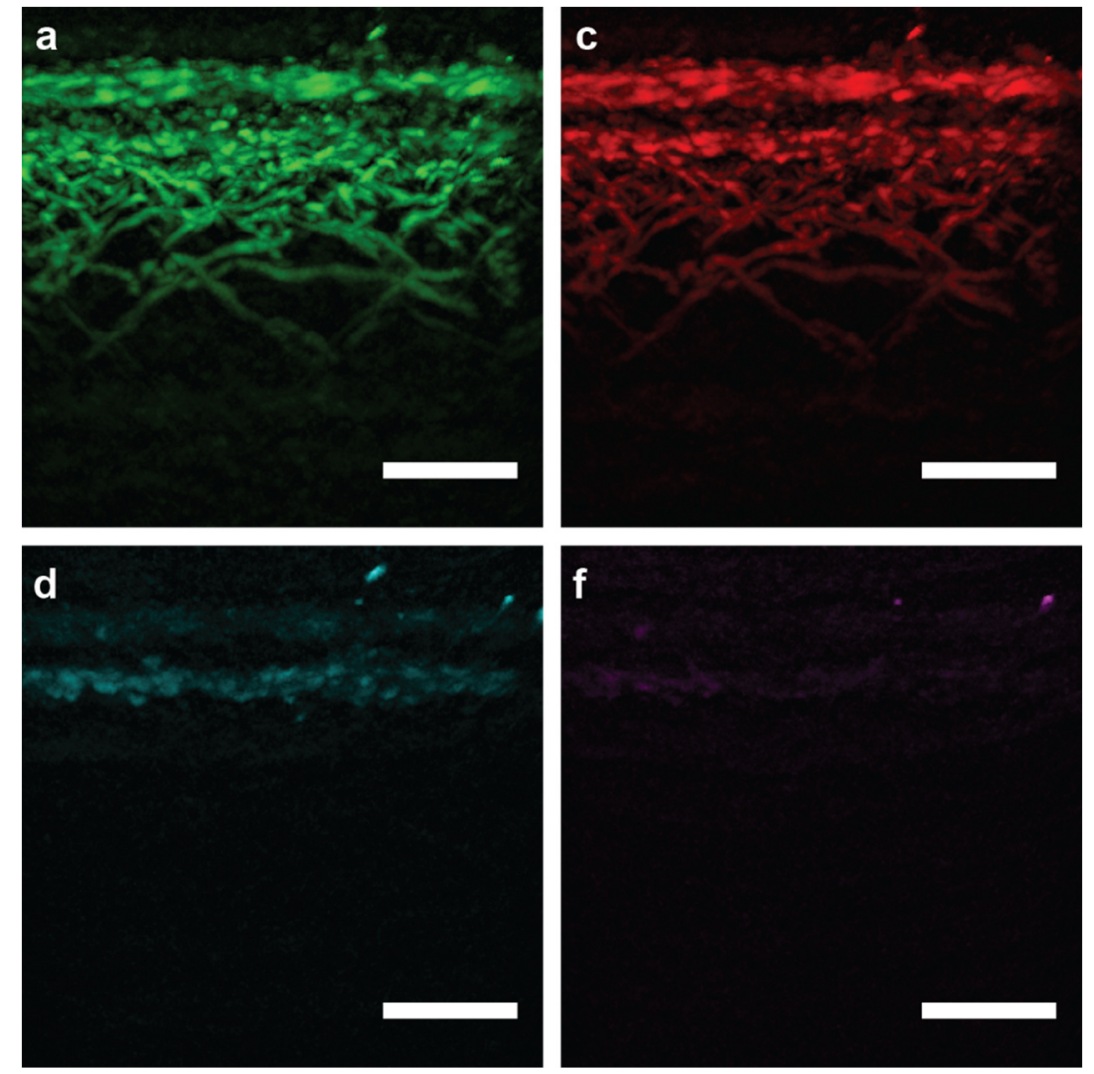

Supplementary Figure 3. Cross-sectional in-vivo images of human skin using FCOT at four wavelengths. a presents the human skin at $445 \mathrm{~nm}$ and $\boldsymbol{b}$ at $465 \mathrm{~nm}$. Both wavelengths were able to detect melanin and blood vessels located deeper in the skin. $\boldsymbol{c}$ presents the skin at 638nm where only weak contrast from melanin is detected. $\boldsymbol{d}$ shows the skin as detected at 808nm where blood and melanin absorption are weak to generate a detectable OA signal. Green is $445 \mathrm{~nm}$, Red is $465 \mathrm{~nm}$, Magenta is $638 \mathrm{~nm}$, Cyan is $808 \mathrm{~nm}$, scale bar $1 \mathrm{~mm}$ in the horizontal direction, vertical range is $1 \mathrm{~mm}$ total. 


\section{Human Skin Experiment}

548

549

550

551

552

553 Since we know that $S_{r}(t)$ contains a periodic signal with additive noise we can restrict it to the

One of the authors volunteered for this study to have their hand imaged. After consultation with the TUM Ethics Commission, no formal ethics approval was necessary. Informed consent from the participant was obtained and archived.

\section{Frequency Comb Algorithm - Formula derivation}

In FCOT the recorded signal over $\mathrm{N}_{\mathrm{p}}$ pulses is $S_{r}(t)=S(t)+n(t)$, where $S(t)$ is the periodic OA signal and $n(t)$ the additive white Gaussian noise. Since $S(t)$ is periodic, we can express its k-th period as $S_{r, k}(t)=S_{r}(t) * \delta(t+k T)$, where $*$ denotes the convolution with the delta function. From Fourier theory we know that

$$
S(\omega)=S_{0}(\omega) \sum_{m=-\infty}^{\infty} \delta\left(\omega-m \omega_{0}\right)
$$

with $\omega_{0}=2 \pi / T$ and $T$ the period of $S_{r}(t)$. This equation indicates that the Fourier transform of a periodic signal is the Fourier transform over one period of the periodic signal multiplied by a series of deltas that confine the Fourier transform of the periodic signal to the harmonics of its repetition rate.

We can now calculate the averaged signal

$$
S_{a}(t)=\frac{1}{N_{p}} \sum_{k=1}^{N_{p}} S_{r, k}(t)=\frac{1}{N_{p}} \sum_{k=1}^{N_{p}} S_{r}(t) * \delta(t+k T)
$$

by shifting the recorded signal back by a multiple of the period each time and summing, with $t$ being $0<t<T$. We can now apply the Fourier transform in equation (2):

$$
S_{a}(\omega)=\frac{1}{N_{p}} \sum_{k=1}^{N_{p}} S_{r}(\omega) e^{j k \omega T}=\frac{1}{N_{p}} S_{r}(\omega) \sum_{k=1}^{N_{p}} e^{j k \omega T}
$$

harmonics of the periodic signal, i.e. multiply equation (3) by $\sum_{m=-\infty}^{\infty} \delta\left(\omega-m \omega_{0}\right)$ : 


$$
S_{a}\left(m \omega_{0}\right)=\frac{1}{N_{p}} S_{r}(\omega) \sum_{k=1}^{N_{p}} e^{j k \omega T} \sum_{m=-\infty}^{\infty} \delta\left(\omega-m \omega_{0}\right) .
$$

However, $e^{i k m \omega_{0} T}=e^{i k m 2 \pi}=1$ for every integer $k$ and $m$. Therefore, the sum of the exponentials is $N_{p}$ and equation (4) becomes

$$
S_{a}\left(m \omega_{0}\right)=S_{r}(\omega) \sum_{m=-\infty}^{\infty} \delta\left(\omega-m \omega_{0}\right)
$$

This equation tells us that the Fourier transform of the averaged signal is the Fourier transform of the recorded signal confined to the harmonics of its repetition rate. We can further expand the recorded signal to obtain

$$
S_{a}\left(m \omega_{0}\right)=S(\omega) \sum_{m=-\infty}^{\infty} \delta\left(\omega-m \omega_{0}\right)+n(\omega) \sum_{m=-\infty}^{\infty} \delta\left(\omega-m \omega_{0}\right)=S_{0}\left(m \omega_{0}\right)+n\left(m \omega_{0}\right),
$$

which tells us that the Fourier transform of the averaged signal is the Fourier transform of the first period of the periodic signal, what we wanted to recover, plus the Fourier transform of the white Gaussian noise confined to the harmonics of the repetition rate of the periodic signal. Since it is not periodic, the noise is significantly suppressed with its power reduced by sqrt(Np).

\section{Modulation parameters for the FCOT - Formula Derivation}

In this work, the FCOT signal is acquired in the Time Domain using a high speed digitizer. In FCOT the $\mathrm{N}$ wavelengths have a different repetition rate, with a small frequency shift $\delta f<<f_{\text {rep }}$. For each wavelength, we follow the same FCOT signal processing algorithm for a single wavelength, choose only the harmonics of the laser repetition rate and apply the inverse Fourier Transform to recover the TD signal. For the case of multiple wavelengths all harmonics from any laser repetition rate should be accurately resolved from all harmonics of each other's laser repetition rate. If any of the harmonics are not well-resolved, there will be a cross-talk between the signals from different wavelengths. Therefore, the choice of the number of pulses in the pulse train and the frequency shift $\delta f$ is critical to be able to separate the signal from each wavelength. In the following we present the analytical 
derivation of the formulas that impose the limits on the number of pulses in the pulse train for each wavelength and the frequency shift.

The first wavelength has a reference repetition rate $f_{\text {rep1, }}$, the second wavelength a repetition rate $\mathrm{f}_{\text {rep } 2}=\mathrm{f}_{\text {rep } 1}+\delta f$ and the $N^{\text {th }}$ wavelength a repetition rate $\mathrm{f}_{\text {repN }}=\mathrm{f}_{\text {rep } 1}+(\mathrm{N}-1) * \delta f$. The pulse train of the first laser contains $\mathrm{N}_{\text {pulses }}$ pulses; this leads to $t_{\text {acq }}=N_{\text {pulses }} / f_{\text {rep1 }}$ acquisition time. For each AScan we apply a Fourier Transform on the data to get the discrete frequency peaks in the Fourier Domain. In Fourier Domain the maximum frequency resolution will be $d f=1 / t_{\text {acq. }}$. The Ultrasound Transducer (UST) exhibits a specific detection frequency range from $f_{\text {low }}$ to $f_{\text {high, }}$ taken as the $-6 d B$ cut-off point of its frequency response.

For the reference wavelength, laser 1 , we need to find the harmonics that are located and the limits of the UST detection range, i.e. the first $k_{s}$ for which, $k_{s} * f_{\text {rep } 1}>f_{\text {low, }}$, and the last $k_{f}$ for which, $k_{f} * f_{r e p 1}<f_{\text {high. }}$. This happens for $k_{s}=\operatorname{ceil}\left(f_{\text {low }} / f_{\text {rep } 1}\right)$ and $k_{f}=f l o o r\left(f_{\text {high }} / f_{\text {rep } 1}\right)$, where floor $(x)$ rounds $x$ to the nearest integer smaller than or equal to $\mathrm{x}$ and ceil( $\mathrm{x})$ rounds $\mathrm{x}$ to the nearest integer greater or equal than $\mathrm{x}$.

At the lower end of the UST detection range, the harmonic $k_{s}$ of laser 1 will be very close to the $k_{s}$ harmonic of laser 2, i.e. $\Delta f=k_{s} * f_{\text {rep } 2}-k_{s} * f_{\text {rep } 1}=k_{s} *\left(f_{\text {rep } 1}+\delta f\right)-k_{s} * f_{\text {rep } 1}=k s * \delta f$. To be able to resolve these two frequencies $\Delta f>d f$, i.e. $\delta f>d f / k_{s}$. This condition applies a minimum limit to the frequency shift $\delta f$, with $\delta f_{\min }=d f / k_{s}$.

At the higher end of the UST detection range, the $\mathrm{k}_{\mathrm{f}}$ harmonic of laser 1 will be very close to the $\mathrm{k}_{\mathrm{f}}-1$ harmonic of laser $N$, i.e. $\Delta f=k_{f} * f_{\text {rep } 1}-\left(k_{f}-1\right) * f_{\text {repN }}=k_{f} * f_{\text {rep } 1}-\left(k_{f}-1\right) *\left[f_{\text {rep } 1}+(N-1) * \delta f\right]=f_{\text {rep } 1}-\left(k_{f}-1\right) *(N-$ $1)^{*} \delta f$. To be able to resolve these two frequencies $\Delta f>d f$, i.e. $\delta f<\left(f_{r e p 1}-d f\right) /\left[\left(k_{f}-1\right)^{*}(N-1)\right]$. This condition applies a maximum limit to the frequency shift $\delta f$, with $\delta f_{\max }=\left(f_{\text {rep } 1}-d f\right) /\left[\left(k_{f}-1\right) *(N-1)\right]$.

We observe that as $\mathrm{N}_{\text {pulses }}$ decreases, the acquisition time decreases and the frequency resolution $\mathrm{df}$ increases. Therefore, as $N_{\text {pulses }}$ decreases, $\delta f_{\min }$ increases and $\delta f_{\max }$ decreases. As $\delta f_{\min }$ should be smaller than $\delta f_{\max }$, this imposes a minimum limit for $N_{\text {pulses }}$ in the case that $\delta f_{\min }=\delta f_{\max }$, $\mathrm{N}_{\text {pulses_min }}=$ ceil $\left\{\left[\left(\mathrm{k}_{\mathrm{f}}-1\right) *(\mathrm{~N}-1)+\mathrm{ks}\right] / \mathrm{k}_{\mathrm{s}}\right\}$. Any number of pulses in the pulse train larger than $\mathrm{N}_{\text {pulses_min }}$ is 
valid and allows for a valid definition of a range for the small frequency shift $\delta f$, between $\delta f_{\min }$ and $\delta f_{\max }$

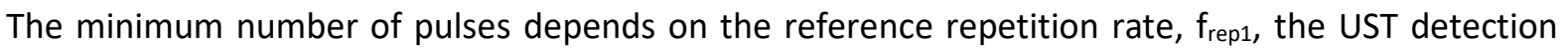
range, $f_{\text {low }}$ and $f_{\text {high, }}$ and the number of wavelengths used, $N$. Once a valid $N_{\text {pulses }}$ is chosen, the range of valid $\delta f^{\prime}$ s then depends on $f_{\text {rep1 }}, f_{\text {low }}, f_{\text {high }}, N$ and $N_{\text {pulses }}$ and a valid frequency shift can be chosen. Following the above, for the bandwidth of the UST that we used $(22-78 \mathrm{MHz})$ we use a reference repetition rate of $f_{\text {rep1 }}=200 \mathrm{KHz}$ and 4 wavelengths. This results in a minimum possible $\mathrm{N}_{\text {pulses }}$ equal to 12. Since we are using laser diodes that usually provide low $S N R$, we chose $N_{\text {pulses }}=100$ to increase the SNR through averaging. For this number of pulses, $\delta f$ can be in the range between $18.2 \mathrm{~Hz}$ and 169.7 $\mathrm{Hz}$. For the frequency shift we chose $\delta f=125 \mathrm{~Hz}$, which gives the base repetition rate for each laser as $f_{\text {rep } 1}=200000 \mathrm{~Hz}, f_{\text {rep } 2}=200125 \mathrm{~Hz}, f_{\text {rep } 3}=200250 \mathrm{~Hz}, f_{\text {rep } 4}=200375 \mathrm{~Hz}$. Moreover, the number of pulses in each pulse train is adjusted to fit as many pulses as possible in the acquisition time defined by the reference repetition rate and $N_{\text {pusles1. }}$. In this case, the following $N_{\text {pulses }}$ are chosen for each wavelength respectively, $N_{\text {pulses } 1}=100, N_{\text {pulses } 2}=101, N_{\text {pulses } 3}=101, N_{\text {pulses } 4}=101$.

\section{Wavelength limit in FCOT compared to TD OA}

There is a maximum number of wavelengths that can be pulsed simultaneously in FCOT. In all cases, $\delta f_{\min }$ should be smaller than $\delta f_{\max }$, i.e. $\delta f_{\min }<\delta f_{\max }$, and by substituting their values as calculated above and solving for the maximum number of wavelength $N_{\max }$ we get $N_{\max }=f l o o r\left\{\left[\left(f_{\text {rep }, 1}-d f\right) * k_{s}\right] /\left[\left(k_{f}-1\right) * d f\right]\right.$ $+1\}$. Assuming an $\mathrm{f}_{\mathrm{rep}, 1}=200 \mathrm{kHz}, \mathrm{N}_{\mathrm{p}}=100$ and a $-6 \mathrm{~dB}$ UST bandwidth between 22 and $78 \mathrm{MHz}$ ( $f_{\text {low }}=22 \mathrm{MHz}$ and $f_{\text {high }}=78 \mathrm{MHz}$ ) we get $N_{\max }=28$. For the same parameters in TD OA a repetition rate of $200 \mathrm{kHz}$ results in a total DoV of $7.5 \mathrm{~mm}$ and assuming that we need at least $1.5 \mathrm{~mm}$ DoV for each wavelength no more than $7.5 / 1.5=5$ wavelengths can be multiplexed without compromising SNR or the total acquisition time. 